\title{
Asymmetric Quinoxaline-Based Polymer for High Efficiency Non-Fullerene Solar Cells
}

\author{
YUAN Jun ${ }^{1}$, LIU Ye ${ }^{1}$, ZHU Can ${ }^{1}$, SHEN Ping ${ }^{2}$, WAN Meixiu ${ }^{3}$, FENG Liuliu ${ }^{1}$, ZOU Yingping ${ }^{1,{ }^{*}}$ \\ ${ }^{1}$ College of Chemistry and Chemical Engineering, Central South University, Changsha 410083, P. R. China. \\ ${ }^{2}$ College of Chemistry, Xiangtan University, Xiangtan 411105, Hunan Province, P. R. China. \\ ${ }^{3}$ Institute of New Energy Technology, College of Information and Technology, Jinan University, Guangzhou 510632, P. R. China.
}

\begin{abstract}
Polymer solar cells (PSCs) with bulk heterojunction (BHJ) structures have seen rapid development in recent years. In comparison with their inorganic counterparts, PSCs have some advantages such as low cost, light weight, solution processability, and good mechanical flexibility. However, improvement of the power conversion efficiency (PCE) of PSCs is required for commercial applications. In order to achieve highperformance PSCs, active layers, including donor polymers and acceptors, are very important. Several design principles for conjugated donor polymers in PSCs have emerged, including optimization of the conjugated backbone, side-chains, and substituents. In the past few decades, various

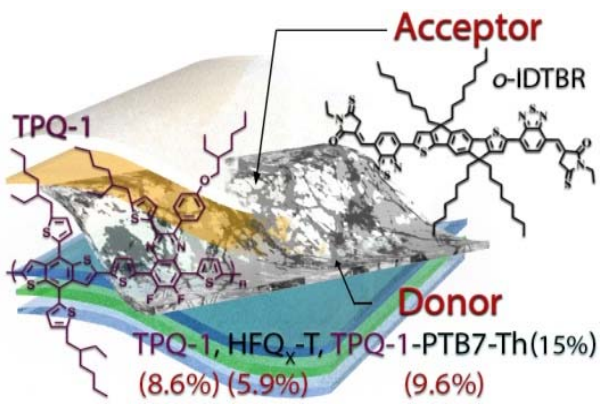
classes of electron-donating polymers have been reported for PSCs. Among them, quinoxaline (Qx) is a unique building block for the construction of different optoelectronic polymers because of its planar, rigid, and conjugated structure. Qx derivatives have proven interesting and have been widely employed in many fields. Qx-based conjugated polymers (or small molecules) can be easily modified to match with ball-like fullerene derivatives such as PCBM ([6,6]-phenyl-C 61 or $\mathrm{C}_{71}$-butyric acid methyl ester) or weak crystalline non-fullerene acceptors such as 2,2'-[[6,6,12,12-tetrakis(4-hexylphenyl)6,12,-dihydrodithieno[2,3-d:2',3'-d']-s-indaceno[1,2-b:5,6-b']dithiophene-2,8-diyl]bis[methylidyne(3-oxo-1H-indene-2,1(3H) -diylidene)]]bispropanedinitrile (ITIC). Herein, we synthesized a Qx-based polymer with asymmetric side-chains (TPQ-1). The molecular weight, optical properties, molecular energy levels, and mobilities of TPQ-1 were investigated. Furthermore, the blend morphologies and photovoltaic properties of TPQ-1 using a strong crystalline non-fullerene (NF) acceptor (oIDTBR) were systematically explored. The photovoltaic performance of TPQ-1 and its symmetric side-chain counterpart, HFQx-T, was compared. The introduction of asymmetric side-chains led to a favorable phase separation when blended with o-IDTBR. As expected, the TPQ-1:0-IDTBR-based devices exhibited a high PCE of $8.6 \%$ after thermal annealing (TA). In contrast, the HFQx-T:o-IDTBR-based devices showed a moderate PCE of 5.7\%, moreover, the PCE was decreased to $4.6 \%$ after TA treatment. More importantly, a low bandgap material, PTB7-Th, was specifically selected as a third component to mix with the TPQ-1:0-IDTBR blend to form highly-efficient ternary PSCs. At an optimal weight ratio (15\%) of PTB7-Th addition, a PCE of $9.6 \%$ was achieved. In the systems that were investigated, TPQ-1 demonstrated significantly better photovoltaic properties than the HFQx-T-based devices. These results indicate that Qx-based polymers with asymmetric side chains have a bright future in photovoltaic devices.
\end{abstract}

Key Words: Non-fullerene solar cells; Asymmetric side chain; Quinoxaline based polymer; Phase separation

Received: February 22, 2018; Revised: March 19, 2018; Accepted: March 20, 2018; Published online: March 22, 2018

*Corresponding author. Email: yingpingzou@csu.edu.cn; Tel.: +86-731-88879616.

The project was supported by the National Natural Science Foundation of China $(51673205,51173206)$ and the Science Fund for Distinguished Young

Scholars of Hunan Province, China (2017JJ1029).

国家自然科学基金(51673205, 51173206)和湖南省杰出青年基金(2017JJ1029)资助项目

(C) Editorial office of Acta Physico-Chimica Sinica 


\title{
基于侧链不对称喹喔啉聚合物的高效非富勒烯太阳电池
}

\author{
袁俊 ${ }^{1}$, 刘晔 ${ }^{1}$, 朱灿 ${ }^{1}$, 沈平 ${ }^{2}$, 万梅秀 ${ }^{3}$, 冯柳柳 ${ }^{1}$, 邹应萍 1 , ${ }^{*}$ \\ 1 中南大学化学化工学院, 长沙 410083 \\ 2 湘潭大学化学学院, 湖南湘潭 411105 \\ 3 暨南大学信息科学技术学院新能源技术研究院, 广州 510632
}

\begin{abstract}
摘要: 喹喔啉衍生物由于合成简单, 易功能化, 成本较低等特点在众多领域都有广泛应用。其自身具有平面刚性结构, 也是构建光电聚合物的重要单体。基于喹喔啉单元的有机分子化学结构和电子结构可修饰性强, 通过骨架、侧链和取代 基等修饰, 易于调控分子的能级和吸光光谱, 因此, 当使用喹喔啉体系的共轭给体与球形富勒烯受体(如PCBM)及弱结 晶性非富勒烯受体(如ITIC)均可表现出优异的光伏性能。在本工作中, 基于结晶性较强的非富勒烯受体(O-IDTBR), 我们 首次制备出侧链不对称喹喔啉(简称：不对称喹咥啉)基聚合物(TPQ-1)与之匹配。相比于侧链对称性哇喔啉(简称：对称 喹咥啉)(HFQx-T)与o-IDTBR组合, “弱结晶给体-强结晶受体” 组合能表现出更佳均匀的相分离尺度, 从而获得更高的 短路电流及能量转换效率。TPQ-1与o-IDTBR 共混后器件效率为 $8.6 \%$, 加入 $15 \%$ 的TB7-Th后, 器件效率达到 $9.6 \%$ 。
\end{abstract}

关键词: 非富勒烯太阳电池; 侧链不对称; 喹喔啉基聚合物; 相分离 中图分类号：0649

\section{1 引言}

有机光伏电池(OPV)因其质量轻、价格低廉、 易加工成大面积柔性器件等优点, 近年来广受学 术界和工业界关注, 成为当下热门研究领域之 - ${ }^{1-3}$ 。有机光伏电池的性能主要取决于活性层对 光的吸收和转换过程, 因此活性层材料的设计及 合成成为研究的关键。当前高性能太阳电池活性 层主要采取给体和受体共混的本体异质结(BHJ) 结构。广泛应用的给体材料一般是具有推-拉(D-A) 结构的低能隙聚合物或小分子, 受体材料则主要 是富勒烯及其衍生物 (PCBM $)^{4-6}$ 。然而, 富勒烯衍 生物吸收窄以及最低未占据分子轨道(LUMO) 能 量低 (与聚合物共混后能量损失较大), 一定程度上 限制了短路电流密度 $\left(J_{\mathrm{sc}}\right)$ 及开路电压 $\left(V_{\mathrm{oc}}\right)$ 的进一 步提高。近几年来, 非富勒烯受体(NFAs)材料的设 计和应用得到了快速发展, 其能量转换效率 (PCEs) 已经可以与富勒烯聚合物太阳电池相媲 美 $7-37$ 。例如, 2015 年, 占肖卫教授研究组 ${ }^{38}$ 率 先报道了 A-D-A 型非富勒烯小分子受体材料 ITIC, 采用 PTB7-Th 作为给体材料, 取得 $6.8 \%$ 的 能量转换效率, 展现了小分子受体具备取代 PCBM 的潜力。李永舫院士研究组 ${ }^{39-41}$ 考察了 ITIC 的吸收光谱及电化学能级后, 将一类与其吸 收互补、能级匹配的聚合物给体材料(J 系列)与 ITIC 衍生物共混, 使其 PCEs 超过 11\%。2016 年, 本研究组采用了一种中带隙(medium bandgap)的 四氟代喹喔啉类聚合物(PffQx-T)与 ITIC 共混, 制 备的聚合物太阳电池获得了 $8.47 \%$ 的 $\mathrm{PCE}^{42,43}$ 。在
此工作基础上, 我们发现对喹喔啉侧链功能化可 有效改善其聚合物薄膜形貌(分子的有序堆积和 聚合物的结晶性)。喹喔啉的优势在于其合成较简 单，易修饰(具有多个活性位点) ${ }^{44-46}$; 因此, 可通 过使用不同侧链修饰的喹喔啉结构来优化聚合物 给体-非富勒烯受体共混膜形貌及能量损失 $\left(E_{\text {loss }}\right)$, 从而深入探索了聚合物结构与性能关系。例如, 六 氟代喹喔啉(HFQx-T)基聚合物与 ITIC 共混, $E_{\text {loss }}$ 可从四氟代喹喔啉的 $0.70 \mathrm{eV}$ 降至 $0.65 \mathrm{eV}$, 因此 PCE 可达 9.4\% ${ }^{47-49}$ 。

在本工作中, 基于对电池薄膜的形貌调控(改 变结晶度), 合成侧链不对称喹喔啉 (以下简称: 不 对称喹喔啉)基聚合物给体(弱结晶性)(TPQ-1, 图 1a)匹配结晶性较强的非富勒烯受体(o-IDTBR, 图 1b)来探索其构效关系 50,51 。与此同时, 我们与侧 链对称性喹喔啉(以下简称: 对称喹喔啉)基聚合物 给体(HFQx-T, 图 1a): o-IDTBR 组合进行对比发 现, “弱结晶性聚合物给体-强结晶性非富勒烯受 体” 组合能发挥更好的光伏性能。形貌测试表明, 使用聚集较弱的无定形聚合物给体, 与聚集较强 的非富勒烯受体共混可有效维持理想的纳米尺度 相分离聚集态结构, 从而实现高效的激子分离和 电荷传输 ${ }^{2,53}$ 。因此, 基于传统的器件结构, TPQ1:o-IDTBR 表现出 $8.6 \%$ 的 PCE, 其中 $V_{\mathrm{oc}}$ 为 0.90 $\mathrm{V}, J_{\mathrm{sc}}$ 为 $14.95 \mathrm{~mA} \cdot \mathrm{cm}^{-2}$ 和填充因子 $(\mathrm{FF})$ 为 $64.3 \%$ 。 而相同条件下 HFQx-T:o-IDTBR 组合器件效率只 有 $4.6 \%$ 。当使用 $15 \%$ 质量比的 PTB7-Th 作为第二 组分给体添加到 TPQ-1:0-IDTBR 体系中, 三元器 
(a)

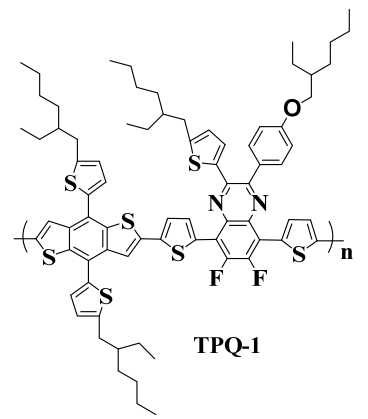

(b)

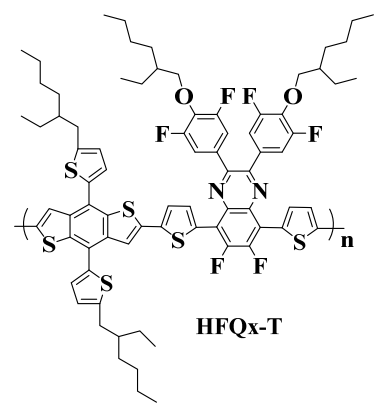

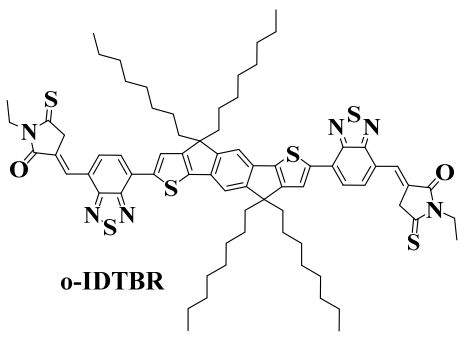

图 1 （a）给体聚合物 TPQ-1 和 HFQx-T; (b) 非富勒烯小分子 o-IDTBR 的化学结构

Fig. 1 The chemical structures (a) donor polymer TPQ-1 and HFQx-T, (b) non-fullerene small molecule acceptor o-IDTBR.

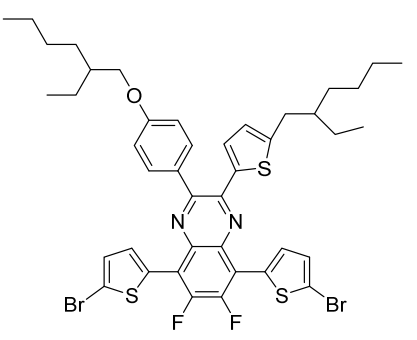

M1

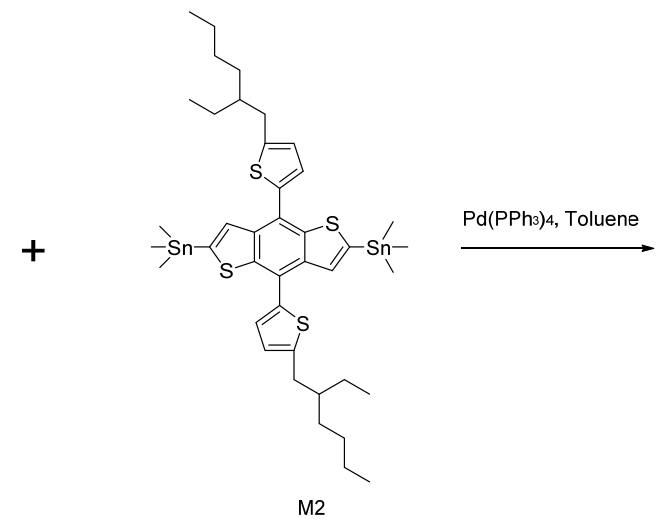

M2

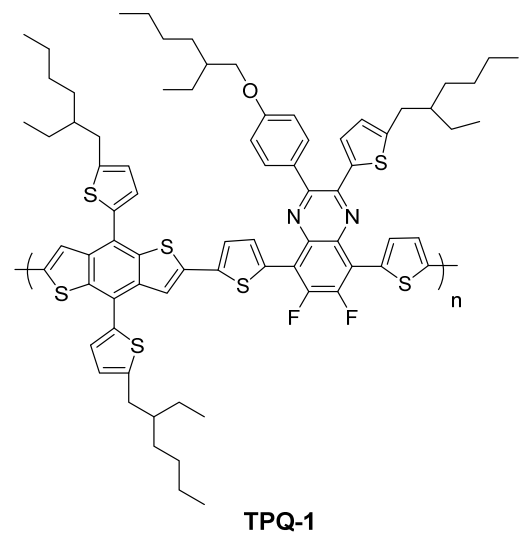

TPQ-1

图 2 聚合物 TPQ-1 合成路线

Fig. 2 Synthetic route of polymer TPQ-1.

件效率最高可达 $9.6 \%$ 。

\section{2 结果与讨论}

\section{$2.1 \mathrm{TPQ}-1$ 的合成及结构表征}

不对称聚合物 TPQ-1 合成路线见图 2。单体 $\mathrm{M} 1$, 通过 NMR 图谱分析确认结构 (见图 S1, Supporting Information)。单体 M1 和单体 M2 通过 Stille 偶联反应得到目标聚合物 TPQ-1。合成的聚 合物依次使用甲醇、正己烷和三氯甲烷抽提纯化, 收集三氯甲烷相, 旋干, 干燥得到目标产物。用高 温凝胶渗透色谱 (GPC) 来测定其分子量及其分布, TPQ-1 的数均分子量 $\left(M_{\mathrm{n}}\right)$ 和多分散系数 (PDI) 分别 为 $15 \mathrm{kDa}$ 和 2.1。聚合物 TPQ-1 的热稳定性通过 热重曲线(TGA)分析表征。在惰性气体保护下, 聚 合物 TPQ-1 具有相对较高的热稳定性, 它的 5\%质 量损失分解温度为 $308{ }^{\circ} \mathrm{C}$ 。

\section{$2.2 \mathrm{TPQ}-1$ 的吸收光谱与能级}

共轭聚合物 TPQ-1 在三氯甲烷溶液和薄膜状 态下的紫外-可见吸收光谱如图 3a, 相应的数据列 于表 1 中。TPQ-1 在溶液状态下显示两个明显的 吸收峰, 一个峰在波长 400-500 nm 处, 这是由于 $\pi-\pi^{*}$ 跃迁引起。另一个峰在 500-700 $\mathrm{nm}$ 处, 是由 给-受体单元电荷转移(ICT) 导致。与溶液相比, 聚 合物薄膜状态的吸收有 $20 \mathrm{~nm}$ 红移, 说明 TPQ-1 在薄膜状态中有略微聚集。聚合物的光学带隙 (bandgap)根据其薄膜吸收边 $(703 \mathrm{~nm}$ )计算为 1.76 $\mathrm{eV}$ 。从图 S2 (Supporting Information)可计算得非 富勒烯小分子 o-IDTBR 光学带隙为 $1.60 \mathrm{eV}$, 可以 与聚合物给体 TPQ-1 形成互补吸收, 这也是实现 宽吸收光谱, 获得高性能光伏器件前提。

为考察材料的最高占据分子轨道(HOMO)与 最低未占分子轨道(LUMO)的能级。我们使用循环 伏安法 $(C V)$ 测定聚合物的电化学性质。循环伏安 曲线测定由 CHI660E 电化学工作站来完成, 以无 水乙腈作为溶剂, $\mathrm{Fc} / \mathrm{Fc}^{+}$作标定, 四丁基六氟磷酸 铵 $\left(\mathrm{TBAPF}_{6}\right)$ 作为电解质, 进行电化学的测定时, 经 过鼓泡排除溶液中的氧。从图 $3 \mathrm{~b}$ 中可得 TPQ-1 的 氧化起始电位和还原起始电位分别为 0.86 和 $-0.97 \mathrm{~V}$ 。相应的 HOMO 和 LUMO 能级通过以下 公式计算得到:

$E_{\mathrm{HOMO} / \mathrm{LUMO}}=-\left(4.8-E_{1 / 2, \mathrm{Fc} / \mathrm{Fc}}{ }^{+}+E_{\text {onset,ox/red }}\right)$ 通过计算可以得到聚合物 PTQ-1 的 $\mathrm{HOMO} /$ 

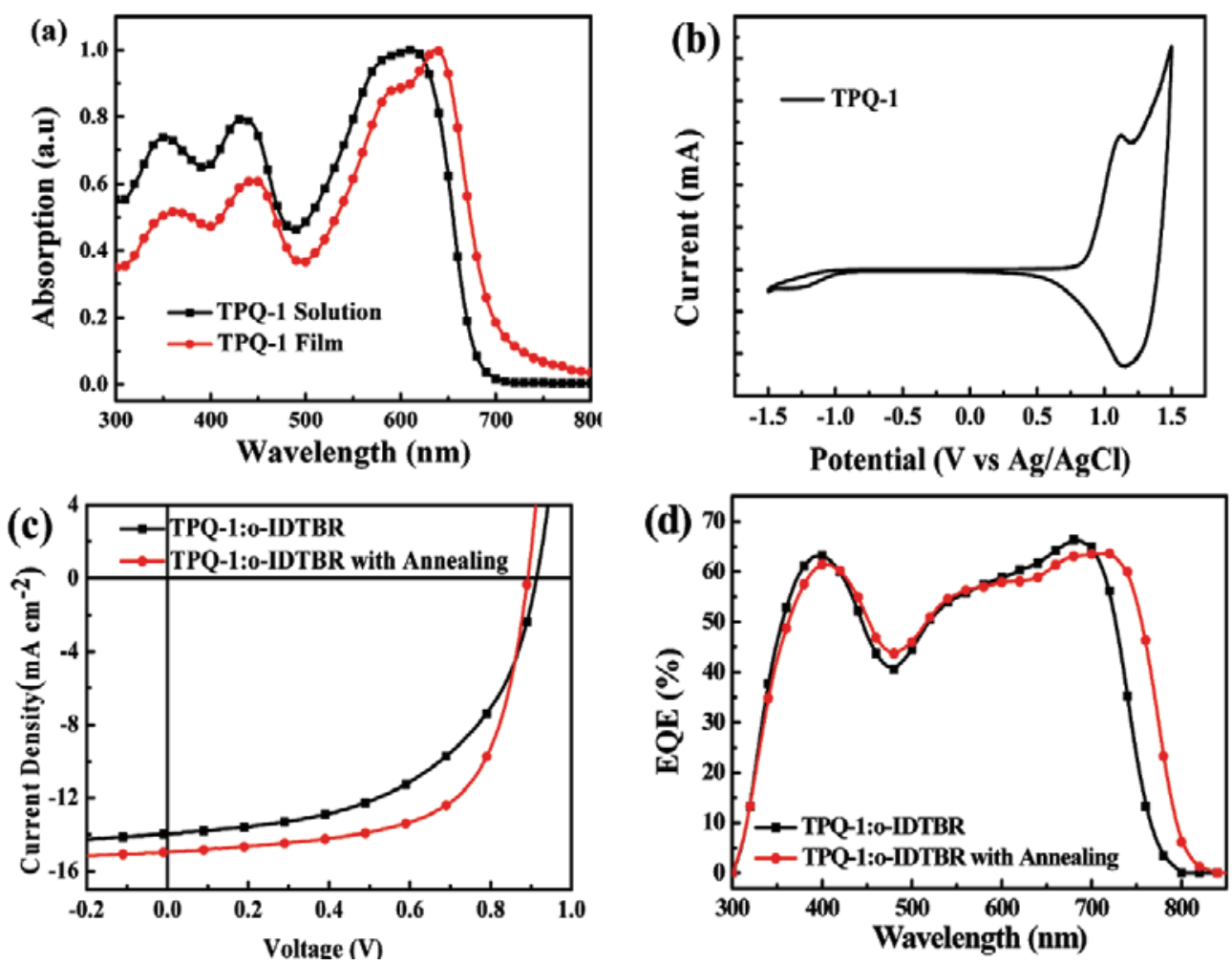

图 3 (a) TPQx-1 在氯仿溶液和固态薄膜中的吸收光谱; (b) TPQx-1 的循环伏安测试曲线; (c) 基于聚合物给体 TPQx-1 和非富勒烯小分子 o-IDTBR 光伏器件的电流密度-电压 $(J-V)$ 特性曲线; (d) 基于聚合物给体 TPQx-1 和 非富勒烯小分子 o-IDTBR 光伏器件的外量子效率(EQE)

Fig. 3 (a) Absorption spectra of TPQx-1 in $\mathrm{CHCl}_{3}$ solution and in a thin film; (b) Cyclic voltammogram of the TPQx-1;

(c) $J-V$ characteristics of the devices based on donor polymer TPQ-1 with non-fullerene small molecular o-IDTBR under illumination of AM 1.5, $100 \mathrm{~mW} \mathrm{~cm}^{-2}$; (d) EQE spectra of the devices based on donor polymers TPQ-1 with non-fullerene small molecular o-IDTBR.

表 1 聚合物 TPQ-1 光学数据和电化学数据

Table 1 Optical and electrochemical properties of TPQ-1.

\begin{tabular}{|c|c|c|c|c|c|c|c|c|c|}
\hline \multirow{3}{*}{ Polymer } & \multicolumn{4}{|c|}{ Absorption spectra } & \multicolumn{5}{|c|}{ Cyclic voltammetry } \\
\hline & \multirow{2}{*}{$\frac{\mathrm{Sol}^{\mathrm{a}}}{\lambda_{\max } / \mathrm{nm}}$} & \multicolumn{3}{|c|}{ Film $^{b}$} & \multicolumn{2}{|c|}{$p$-doping } & \multicolumn{2}{|c|}{$n$-doping } & \\
\hline & & $\lambda_{\max } / \mathrm{nm}$ & $\lambda_{\text {onset }} / \mathrm{nm}$ & $E_{\mathrm{g}}^{\mathrm{opt}} / \mathrm{eV}^{\mathrm{c}}$ & $E_{\mathrm{on}}^{\mathrm{ox}} / \mathrm{V}$ & $E_{\mathrm{HOMO}} \mathrm{d} / \mathrm{eV}$ & $E_{\text {on }}^{\text {red }} / \mathrm{V}$ & $E_{\text {LUMO }} \mathrm{d} / \mathrm{eV}$ & $E_{\mathrm{g}}^{\mathrm{EC}} / \mathrm{eV}$ \\
\hline TPQ-1 & 618 & 638 & 704 & 1.76 & 0.86 & -5.26 & -0.97 & -3.43 & 1.83 \\
\hline
\end{tabular}

${ }^{a}$ Measured in chloroform solution. ${ }^{b}$ Cast from chloroform solution. ${ }^{c}$ Bandgap estimated from the onset wavelength of the optical absorption. ${ }^{\mathrm{d}} E_{\mathrm{HOMO}}=-\left(4.8-E_{1 / 2, \mathrm{Fc} / \mathrm{Fc}}{ }^{+}+E_{\mathrm{ox}}\right)(\mathrm{eV}) ; E_{\mathrm{LUMO}}=-\left(4.8-E_{1 / 2, \mathrm{Fc} / \mathrm{Fc}}{ }^{+}+E_{\mathrm{red}}\right)(\mathrm{eV})$ using $\mathrm{Ag} / \mathrm{AgCl}$ as the reference electrode.

LUMO 能级分别为 -5.26 和 $-3.43 \mathrm{eV}$, 而非富勒烯 受体 o-IDTBR 的 HOMO 与 LUMO 能级分别为 -5.51 和 $-3.88 \mathrm{eV}$ 。结果表明, 给受体之间有足够 的驱动力 $(0.3 \mathrm{eV})$ 促使电荷有效分离。光电化学数 据归纳在表 1 中。

\section{$2.3 \mathrm{TPQ}-1$ 的光伏器件性能}

为了研究共轭聚合物 TPQ-1 的光伏性能, 我 们制备了本体异质结单层器件, 器件结构为 ITO(氧化铟锡玻璃)/PEDOT:PSS(聚(3,4-乙烯基二 氧噻吩)与聚磺酸基苯乙烯的混合物)/TPQ-1:0-
$\operatorname{IDTBR} / \mathrm{PDINO}(\mathrm{N}-$ 功能化的荴酰亚胺 $) / \mathrm{Al}$ 。图 $3 \mathrm{c}$ 为 光伏器件在标准模拟太阳光 $(\mathrm{AM} 1.5 \mathrm{G}, 100$ $\left.\mathrm{mW} \cdot \mathrm{cm}^{-2}\right)$ 下的 $J-V$ 曲线图, 表 2 列出了具体器件 结果。为了优化 TPQ-1 器件性能, 我们通过调整 不同的给受比例, 不同的热退火温度等进行了器 件表征, 优化的器件性能数据总结在表 S1-4 (Supporting Information)中。如图 3c 所示, TPQ1 与 $\mathrm{o}-\mathrm{IDTBR}$ 的质量比为 $1: 1.5$ 为器件的最优比 例, 相应的器件效率为 $6.8 \%, V_{\mathrm{oc}}$ 为 $0.91 \mathrm{~V}, J_{\mathrm{sc}}$ 为 $13.94 \mathrm{~mA} \cdot \mathrm{cm}^{-2}$, FF 为 $53.5 \%$ 。我们使用热退火的 
表 2 基于共轭聚合物给体 TPQ-1 和 o-IDTBR 太阳电池器件的性能参数

Table 2 Photovoltaic parameters for TPQ-1:0-IDTBR devices.

\begin{tabular}{|c|c|c|c|c|c|}
\hline Active layer & $V_{\mathrm{oc}} / \mathrm{V}$ & $J_{\mathrm{sc}} /\left(\mathrm{mA} \mathrm{cm}^{-2}\right)$ & $\mathrm{FF} / \%$ & $\mathrm{PCE}_{\max } / \%$ & Average PCE $/ \%$ \\
\hline TPQ-1:o-IDTBR (1:1.5) ${ }^{\mathrm{a}}$ & 0.91 & 13.94 & 53.5 & 6.8 & 6.6 \\
\hline TPQ-1:o-IDTBR $(1: 1.5)^{\mathrm{b}}$ & 0.90 & 14.95 & 64.3 & 8.6 & 8.5 \\
\hline HFQx-T:o-IDTBR $(1: 1)^{a}$ & 1.09 & 9.85 & 55.0 & 5.9 & 5.7 \\
\hline HFQx-T:o-IDTBR (1:1) ${ }^{\mathrm{b}}$ & 1.02 & 6.91 & 64.7 & 4.6 & 4.5 \\
\hline TPQ-1:PTB7-Th: o-IDTBR ${ }^{b}$ & 0.92 & 16.57 & 63.0 & 9.6 & 9.5 \\
\hline
\end{tabular}

a As-cast film. ${ }^{\mathrm{b}}$ With thermal annealing at $110{ }^{\circ} \mathrm{C}$ for $10 \mathrm{~min}$ and $15 \%$ PTB7-Th content.

方法来优化器件性能, 如表 2 所示, 在 $110{ }^{\circ} \mathrm{C}$ 退 火 $10 \mathrm{~min}$ 时, 达到最优的器件性能, 最高的 PCE 为 $8.6 \%$, 相对于没有热退火的器件, 提升超过 26\%。与基于 TPQ-1 的器件相比, 虽然 HFQx-T 的 器件拥有更高的 $V_{\mathrm{oc}}$ (氟原子降低 HOMO 能级), 然而, HFQx-T 器件 $J_{\mathrm{sc}}$ 却只有 $9.85 \mathrm{~mA} \cdot \mathrm{cm}^{-2}$, 在 热退火后甚至降至 $6.91 \mathrm{~mA} \cdot \mathrm{cm}^{-2}$ (图 $\mathrm{S} 3 \mathrm{a}$, Supporting Information), 这表明分子的堆积行为会 受到分子结构的明显影响 54 。图 $3 \mathrm{~d}$ 为聚合物光伏 器件的外量子效率(EQE)曲线。所有的 $\mathrm{EQE}$ 曲线 在 300-800 $\mathrm{nm}$ 之间表现出了明显的光响应。从图 中可看出, 热退火后的共混薄膜有明显的光谱红 移, 这也部分解释了退火后器件拥有更高的 $J_{\mathrm{sc}}$ 。 基于 $\mathrm{EQE}$ 曲线计算的短路电流和采用 $J-V$ 曲线测 试的短路电流值在误差范围内(小于 $5 \%$ ), 证实了 器件测试结果是准确的。与此同时, 采用空间电荷 限制电流(SCLC)方法对活性层的载流子迁移率进 行了表征, 相应的 $J^{1 / 2}-V$ 特性曲线如图 $\mathrm{S} 4 \mathrm{a}$ (Supporting Information)所示。使用的器件结构为 ITO/PEDOT:PSS/TPQ-1:o-IDTBR $(100 \mathrm{~nm}) / \mathrm{Au}$ 。通 过计算公式算出活性层空穴迁移率在热退火和未 退火下分别为 $6.22 \times 10^{-5}$ 和 $1.77 \times 10^{-5}$ $\mathrm{cm}^{2} \cdot \mathrm{V}^{-1} \cdot \mathrm{s}^{-1}$ 。退火后活性层具有更高的空穴迁移 率, 这与退火后器件具有更高的 $J_{\mathrm{sc}}$ 的结果吻合。

使用三元共混器件是克服其 ShockleyQueisser (SQ)极限, 从而进一步提升器件效率的有 效方法。因此, 我们使用 PTB7-Th 作为第三组分 加入到 TPQ-1:0-IDTBR 活性层中来制备三元共混 有机太阳电池。表 S4 总结了基于不同 PTB7-Th 含 量的器件平均和最好的器件性能。PTB7-Th 的不 同加入量会明显影响器件各个参数。与最优状态 下 TPQ-1/ITDBR 器件相比, 含 $15 \%$ (质量分数) PTB7-Th 的三元共混有机太阳电池展现了更佳优 异的性能: $V_{\mathrm{oc}}$ 从 0.90 增加至 $0.92 \mathrm{~V}, J_{\mathrm{sc}}$ 从 14.95 增加至 $16.57 \mathrm{~mA} \cdot \mathrm{cm}^{-2}$, 器件效率从 $8.6 \%$ 增加到 $9.6 \%$ 。相关器件 $J-V$ 和 $\mathrm{EQE}$ 曲线图显示在图 $\mathrm{S} 4 \mathrm{~b}$ 和 $4 \mathrm{c}$ 中。

\section{4 形貌分析}

BHJ 活性层的形貌对光伏性能有很大影响。 区别于聚合物-富勒烯体系(单个光生电荷通道), 聚合物与非富勒烯体系可生成光生电荷双通道, 即共轭聚合物和非富勒烯受体之间电荷转移依赖 给-受之间分子取向。在理想状态下, 给体和受体 需混合形成一个连续纳米尺寸相。在此, 我们从材 料设计角度出发, 设想通过调节给-受分子扩散速 率来构建互穿网络结构。选用聚集作用较弱的聚 合物 TPQ-1 与聚集能力较强的 o-IDTBR 分子共 混, 利用退火方式来控制分子扩散速率, 从而获得 纳米级互穿网络结构。

如图 4a 所示, 共混薄膜在原子力显微镜 (AFM)下几乎没有很明显相分离结构, 共混薄膜的 表面粗粘度(RMS)为 $2.74 \mathrm{~nm}$ 。当利用 $110^{\circ} \mathrm{C}$ 温度 对上述薄膜退火处理 $10 \mathrm{~min}$ (图 4c), o-IDTBR 自
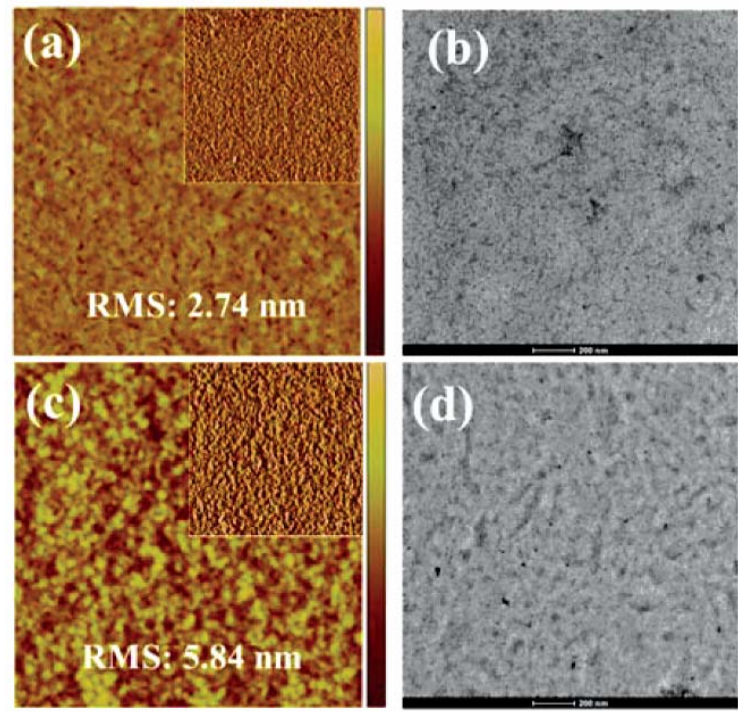

图 4 无热退火/热退火下共混膜的 AFM 图 $(5 \mu \mathrm{m} \times 5 \mu \mathrm{m})$

Fig. 4 AFM images $(5 \mu \mathrm{m} \times \mathbf{5} \boldsymbol{\mu m})$ of blend films.

(a) TPQx-1:o-IDTBR; AFM images of blend as-cast films: (c) TPQx-1:oIDTBR; AFM images of blend films with thermal annealing; TEM images of blend films: (b) TPQx-1:o-IDTBR; TEM images of blend as-cast films: (d) TPQx-1:o-IDTBR; TEM images of blend films with thermal annealing. 
组形成结晶网络骨架, 聚合物 TPQ-1 缓慢结晶, 最终形成比较良好的互穿网络结构, 其 RMS 值变 为 $5.84 \mathrm{~nm}$ 。相反, 基于 HFQx-T:o-IDTBR 组合器 件的膜退火前后的粗䊁度比较大 $(\mathrm{RMS}=5.86 \mathrm{~nm}$ 和 $6.39 \mathrm{~nm}$ ) (图 S5a、c, Supporting Information)。 大的相分离尺寸会导致电荷载流子聚集和电荷复 合, 从而影响器件 $J_{\mathrm{sc}}$ 。相同的实验现象可从透射 电镜(TEM)测试中得到。和 TPQ-1:o-IDTBR 膜相 相比, HFQx-T:o-IDTBR 显示了明显的相分离和较 粗糙的膜相, 这将限制激子的有效分离, 从而降低 器件性能。

\section{3 结论}

在此工作中, 基于结晶性较强的非富勒烯受 体(o-IDTBR), 我们首次制备了侧链不对称喹喔啉 基聚合物(TPQ- 1 )与之匹配, 共混后器件效率为 $8.6 \%$, 加入 $15 \%$ 的 TB7-Th后, 器件效率达到 $9.6 \%$ 。 实验表明, 这种 “弱结晶给体-强结晶受体” 组合 可以有效维持理想的纳米尺度相分离聚集态结 构, 从而实现高效的激子分离和电荷传输。相比于 侧链对称性喹喔啉(HFQ x-T) 与 o-IDTBR组合, “弱 结晶给体-强结晶受体” 组合可通过热退火方式有 效调节给-受分子扩散速率从而导致更加均匀的 相分离尺度。这也为我们从材料合成角度来理性 调控有机太阳电池性能提供了设计思路。

致谢: 通讯作者 2005-2008 年在中国科学院化学研究所李永 舫院士门下攻读博士学位, 非常感谢导师在这期间和之后对 自己研究工作和生活的帮助和大力支持!

Supporting Information: available free of charge via the internet at http://www.whxb.pku.edu.cn.

\section{References}

(1) Li, Y. Acc. Chem. Res. 2012, 45, 723. doi: 10.1021/ar2002446

(2) Lu, L.; Zheng, T.; Wu, Q.; Schneider, A. M.; Zhao, D.; Yu, L. Chem. Rev. 2015, 115, 12666. doi: 10.1021/acs.chemrev.5b00098

(3) Li, G.; Shrotriya, V.; Huang, J.; Yao, Y.; Moriarty, T.; Emery, K.; Yang, Y. Nat. Mater. 2005, 4, 864. doi: 10.1038/nmat 1500

(4) Zhou, H.; Yang, L.; You, W. Macromolecules 2012, 45, 607. doi: $10.1021 / \mathrm{ma} 201648 \mathrm{t}$

(5) Lai, Y. Y.; Cheng, Y. J.; Hsu, C. S. Energy Environ. Sci. 2014, 7 , 1866. doi: 10.1039/C3EE43080D

(6) Günes, S.; Neugebauer, H.; Sariciftci, N. S. Chem. Rev. 2007, 107, 1324. doi: $10.1021 / \mathrm{cr} 050149 \mathrm{z}$

(7) Fu, Y.; Wang, F.; Zhang, Y.; Fang, X.; Lai, W.; Huang, W. Acta
Chim. Sin. 2014, 72, 158. doi: 10.6023/A13111142

(8) Xiao, Z.; Jia, X.; Ding, L. Sci. Bull. 2017, 62, 1562. doi: 10.1016/j.scib.2017.11.003

(9) Dou, C.; Liu, J.; Wang, L. Sci. China Chem. 2017, 60, 450. doi: 10.1007/s11426-016-0503-x

(10) Deng, D.; Zhang, Y.; Zhang, J.; Wang, Z.; Zhu, L.; Fang, J.; Xia, B.; Wang, Z.; Lu, K.; Ma, W.; et al. Nat. Commun. 2016, 7, 13740. doi: 10.1038/ncomms 13740

(11) Zhao, W.; Li, S.; Yao, H.; Zhang, S.; Zhang, Y.; Yang, B.; Hou, J. J. Am. Chem. Soc. 2017, 139, 7148. doi: $10.1021 /$ jacs. $7 \mathrm{~b} 02677$

(12) Wu, Q.; Zhao, D.; Schneider, A. M.; Chen, W.; Yu, L. J. Am. Chem. Soc. 2016, 138, 7248. doi: 10.1021/jacs.6b03562

(13) Meng, D.; Fu, H.; Xiao, C.; Meng, X.; Winands, T.; Ma, W.; Wei, W.; Fan, B.; Huo, L.; Doltsinis, N. L.; et al. J. Am. Chem. Soc. 2016, 138, 10184. doi: 10.1021/jacs.6b04368

(14) Liu, Y.; Zhang, Z.; Feng, S.; Li, M.; Wu, L.; Hou, R.; Xu, X.; Chen, X.; Bo, Z. J. Am. Chem. Soc. 2017, 139, 3356. doi: $10.1021 /$ jacs. $7 b 00566$

(15) Lin, Y.; Zhao, F.; He, Q.; Huo, L.; Wu, Y.; Parker, T. C.; Ma, W.; Sun, Y.; Wang, C.; Zhu, D.; et al. J. Am. Chem. Soc. 2016, 138, 4955. doi: 10.1021/jacs.6b02004

(16) Li, Y.; Lin, J. D.; Che, X.; Qu, Y.; Liu, F.; Liao, L. S.; Forrest, S. R. J. Am. Chem. Soc. 2017, 139, 17114. doi: $10.1021 /$ jacs.7b11278

(17) Kan, B.; Feng, H.; Wan, X.; Liu, F.; Ke, X.; Wang, Y.; Wang, Y.; Zhang, H.; Li, C.; Hou, J.; et al. J. Am. Chem. Soc. 2017, 139, 4929. doi: 10.1021/jacs.7b01170

(18) Liu, Y.; Liu, J.; Zhang, L.; Fang, J.; Zhang, W.; Liu, Z. Chin. J. Org. Chem. 2014, 34, 1021. doi: 10.6023/cjoc201311041

(19) Ma, Y.; Zhang, M.; Tang, Y.; Ma, W.; Zheng, Q. Chem. Mater. 2017, 29, 9775. doi: 10.1021/acs.chemmater.7b03770

(20) Park, G. E.; Choi, S.; Park, S. Y.; Lee, D. H.; Cho, M. J.; Choi, D. H. Adv. Energy Mater. 2017, 7, 1700566. doi: 10.1002/aenm.201700566

(21) Zhang, K.; Gao, K.; Xia, R.; Wu, Z.; Sun, C.; Cao, J.; Qian, L.; Li, W.; Liu, S.; Huang, F.; et al. Adv. Mater. 2016, 28 , 4817. doi: 10.1002/adma.201506270

(22) Zhang, G.; Yang, G.; Yan, H.; Kim, J. H.; Ade, H.; Wu, W.; Xu, X.; Duan, Y.; Peng, Q. Adv. Mater. 2017, 29, 1606054. doi: 10.1002/adma.201606054

(23) Xu, S. J.; Zhou, Z.; Liu, W.; Zhang, Z.; Liu, F.; Yan, H.; Zhu, X. Adv. Mater. 2017, 29, 1704510. doi: 10.1002/adma.201704510

(24) Lin, Y.; Zhao, F.; Wu, Y.; Chen, K.; Xia, Y.; Li, G.; Prasad, S. K.; Zhu, J.; Huo, L.; Bin, H.; et al. Adv. Mater. 2017, 29, 
1604155. doi: 10.1002/adma.201604155

(25) Cheng, P.; Wang, R.; Zhu, J.; Huang, W.; Chang, S. Y.; Meng, L.; Sun, P.; Cheng, H. W.; Qin, M.; Zhu, C.; et al. Adv. Mater. 2018, 30, 1705243. doi: 10.1002/adma.201705243

(26) Fei, Z.; Eisner, F. D.; Jiao, X.; Azzouzi, M.; Rohr, J. A.; Han, Y.; Shahid, M.; Chesman, A. S. R.; Easton, C. D.; McNeill, C. R.; et al. Adv. Mater. 2018, 30, 1705209. doi: 10.1002/adma.201705209

(27) Huang, W.; Cheng, P.; Yang, Y. M.; Li, G.; Yang, Y. Adv. Mater. 2018, 30, 1705706. doi: 10.1002/adma.201705706

(28) Liu, D.; Wang, J.; Gu, C.; Li, Y.; Bao, X.; Yang, R. $A d v$. Mater. 2018, 30, 1705870. doi: 10.1002/adma.201705870

(29) Luo, Z.; Bin, H.; Liu, T.; Zhang, Z. G.; Yang, Y.; Zhong, C.; Qiu, B.; Li, G.; Gao, W.; Xie, D.; et al. Adv. Mater. 2018, 30, 1706124. doi: 10.1002/adma.201706124

(30) Zhu, J.; Ke, Z.; Zhang, Q.; Wang, J.; Dai, S.; Wu, Y.; Xu, Y.; Lin, Y.; Ma, W.; You, W.; et al. Adv. Mater. 2018, 30, 1704713. doi: 10.1002/adma.201704713

(31) Qiu, B.; Xue, L.; Yang, Y.; Bin, H.; Zhang, Y.; Zhang, C.; Xiao, M.; Park, K.; Morrison, W.; Zhang, Z. G.; et al. Chem. Mater. 2017, 29, 7543. doi: 10.1021/acs.chemmater.7b02536

(32) Feng, G.; Li, J.; Colberts, F. J. M.; Li, M.; Zhang, J.; Yang, F.; Jin, Y.; Zhang, F.; Janssen, R. A. J.; Li, C.; et al. J. Am. Chem. Soc. 2017, 139, 18647. doi: 10.1021/jacs.7b10499

(33) Yao, Z.; Liao, X.; Gao, K.; Lin, F.; Xu, X.; Shi, X.; Zuo, L.; Liu, F.; Chen, Y.; Jen, A. K. J. Am. Chem. Soc. 2018, 140, 2054. doi: $10.1021 /$ jacs.7b13239

(34) Fan, B.; Ying, L.; Wang, Z.; He, B.; Jiang, X. F.; Huang, F.; Cao, Y. Energy Environ. Sci. 2017, 10, 1243. doi: 10.1039/C7EE00619E

(35) Zhang, X.; Zhan, C.; Yao, J. Chem. Mater. 2015, 27, 166. doi: $10.1021 / \mathrm{cm} 504140 \mathrm{c}$

(36) Guo, B.; Li, W.; Guo, X.; Meng, X.; Ma, W.; Zhang, M.; Li, Y. Adv. Mater. 2017, 29, 1702291. doi: 10.1002/adma.201702291

(37) Duan, Y.; Xu, X.; Yan, H.; Wu, W.; Li, Z.; Peng, Q. Adv. Mater. 2017, 29, 1605115. doi: 10.1002/adma.201605115

(38) Lin, Y.; Wang, J.; Zhang, Z. G.; Bai, H.; Li, Y.; Zhu, D.; Zhan, X. Adv. Mater. 2015, 27, 1170. doi: 10.1002/adma.201404317

(39) Bin, H.; Zhang, Z. G.; Gao, L.; Chen, S.; Zhong, L.; Xue, L.; Yang, C.; Li, Y. J. Am. Chem. Soc., 2016, 138, 4657. doi: $10.1021 /$ jacs.6b01744

(40) Bin, H.; Gao, L.; Zhang, Z. G.; Yang, Y.; Zhang, Y.; Zhang,
C.; Chen, S.; Xue, L.; Yang, C.; Xiao, M.; et al. Nat. Commun. 2016, 7, 13651. doi: 10.1038/ncomms 13651

(41) Xue, L.; Yang, Y.; Xu, J.; Zhang, C.; Bin, H.; Zhang, Z. G.; Qiu, B.; Li, X.; Sun, C.; Gao, L.; et al. Adv. Mater. 2017, 29, 1703344. doi: 10.1002/adma.201703344

(42) Yuan, J.; Qiu, L.; Zhang, Z.; Li, Y.; He, Y.; Jiang, L.; Zou, Y. Chem. Commun. 2016, 52, 6881. doi: 10.1039/C6CC01771A

(43) Yuan, J.; Qiu, L.; Zhang, Z. G.; Li, Y.; Chen, Y.; Zou, Y. Nano Energy 2016, 30, 312. doi: 10.1016/j.nanoen.2016.10.008

(44) Yuan, J.; Ouyang, J.; Cimrová, V.; Leclerc, M.; Najari, A.; Zou, Y. J. Mater. Chem. C 2017, 5, 1858. doi: 10.1039/C6TC05381E

(45) Gedefaw, D.; Prosa, M.; Bolognesi, M.; Seri, M.; Andersson, M. R. Adv. Energy Mater. 2017, 7, 1700575. doi: 10.1002/aenm.201700575

(46) Liu, M.; Gao, Y.; Zhang, Y.; Liu, Z.; Zhao, L. Polym. Chem. 2017, 8, 4613. doi: 10.1039/C7PY00850C

(47) Xu, S.; Feng, L.; Yuan, J.; Zhang, Z. G.; Li, Y.; Peng, H.; Zou, Y. ACS Appl. Mater. Interfaces 2017, 9, 18816. doi: 10.1021/acsami.7b03947

(48) Zhang, Z.; Feng, L.; Xu, S.; Yuan, J.; Zhang, Z. G.; Peng, H.; Li, Y.; Zou, Y. J. Mater. Chem. A 2017, 5, 11286. doi: 10.1039/C7TA02486J

(49) Zhang, Z.; Feng, L.; Xu, S.; Liu, Y.; Peng, H.; Zhang, Z. G.; Li, Y.; Zou, Y. Adv. Sci. 2017, 4, 1700152. doi: 10.1002/advs.201700152

(50) Baran, D.; Ashraf, R. S.; Hanifi, D. A.; Abdelsamie, M.; Gasparini, N.; Rohr, J. A.; Holliday, S.; Wadsworth, A.; Lockett, S.; Neophytou, M.; et al. Nat. Mater. 2017, 16, 363. doi: $10.1038 /$ nmat4797

(51) Jia, B.; Wu, Y.; Zhao, F.; Yan, C.; Zhu, S.; Cheng, P.; Mai, J.; Lau, T. K.; Lu, X.; Su, C. J.; et al. Sci. China Chem. 2017, 60, 257. doi: 10.1007/s11426-016-0336-6

(52) Feng, S.; Zhang, C.; Liu, Y.; Bi, Z.; Zhang, Z.; Xu, X.; Ma, W.; Bo, Z. Adv. Mater. 2017, 29, 1703527. doi: 10.1002/adma.201703527

(53) Kouijzer, S.; Michels, J. J.; van den Berg, M.; Gevaerts, V. S.; Turbiez, M.; Wienk, M. M.; Janssen, R. A. J. Am. Chem. Soc. 2013, 135, 12057. doi: 10.1021/ja405493j

(54) Li, Z.; Jiang, K.; Yang, G.; Lai, J. Y.; Ma, T.; Zhao, J.; Ma, W.; Yan, H. Nat. Commun. 2016, 7, 13094. doi: $10.1038 /$ ncomms 13094 